\title{
Pulmonary function and radiological features 4 months after COVID-19: first results from the national prospective observational Swiss COVID-19 lung study
}

\author{
Sabina A. Guler (1) 1 , Lukas Ebner², Catherine Aubry-Beigelman ${ }^{3}$, \\ Pierre-Olivier Bridevaux ${ }^{4}$, Martin Brutsche ${ }^{5}$, Christian Clarenbach (10 ${ }^{6}$, \\ Christian Garzoni ${ }^{7,8}$, Thomas K. Geiser ${ }^{1,9}$, Alexandra Lenoir (10 ${ }^{10}$, \\ Marco Mancinetti ${ }^{11}$, Bruno Naccini ${ }^{12}$, Sebastian R. Ott ${ }^{13}$, Lise Piquilloud ${ }^{14}$ \\ Maura Prella ${ }^{10}$, Yok-Ai Que ${ }^{15}$, Paula M. Soccal ${ }^{16}$, Christophe von Garnier $^{10}$ and \\ Manuela Funke-Chambour (10) ${ }^{1,9}$
}

@ERSpublications

COVID-19 pulmonary sequelae are unknown. The Swiss COVID-19 lung study reports on initial follow-up findings. Severe or critical COVID-19 was associated with significant functional impairment and radiological abnormalities after 4 months. https://bit.ly/34sNVvi

Cite this article as: Guler SA, Ebner L, Aubry-Beigelman C, et al. Pulmonary function and radiological features 4 months after COVID-19: first results from the national prospective observational Swiss COVID19 lung study. Eur Respir J 2021; 57: 2003690 [https://doi.org/10.1183/13993003.03690-2020].

\begin{abstract}
Background: The infectious coronavirus disease 2019 (COVID-19) pandemic is an ongoing global healthcare challenge. Up to one-third of hospitalised patients develop severe pulmonary complications and acute respiratory distress syndrome. Pulmonary outcomes following COVID-19 are unknown.

Methods: The Swiss COVID-19 lung study is a multicentre prospective cohort investigating pulmonary sequelae of COVID-19. We report on initial follow-up 4 months after mild/moderate or severe/critical COVID-19 according to the World Health Organization severity classification.

Results: 113 COVID-19 survivors were included (mild/moderate $n=47$, severe/critical $n=66$ ). We confirmed several comorbidities as risk factors for severe/critical disease. Severe/critical disease was associated with impaired pulmonary function, i.e. diffusing capacity of the lung for carbon monoxide $\left(D_{\text {LCO }}\right) \%$ predicted, reduced 6-min walk distance (6MWD) and exercise-induced oxygen desaturation. After adjustment for potential confounding by age, sex and body mass index (BMI), patients after severe/ critical COVID-19 had a $D_{\text {LCO }} 20.9 \%$ pred (95\% CI $12.4-29.4 \%$ pred, p=0.01) lower at follow-up. $D_{\text {LCO }} \%$ pred was the strongest independent factor associated with previous severe/critical disease when age, sex, BMI, 6MWD and minimal peripheral oxygen saturation at exercise were included in the multivariable model (adjusted odds ratio per $10 \%$ predicted $0.59,95 \%$ CI $0.37-0.87 ; p=0.01$ ). Mosaic hypoattenuation on chest computed tomography at follow-up was significantly associated with previous severe/critical COVID-19 including adjustment for age and sex (adjusted OR 11.7, 95\% CI 1.7-239; p=0.03).

Conclusions: 4 months after severe acute respiratory syndrome coronavirus 2 infection, severe/critical COVID-19 was associated with significant functional and radiological abnormalities, potentially due to small-airway and lung parenchymal disease. A systematic follow-up for survivors needs to be evaluated to optimise care for patients recovering from COVID-19.
\end{abstract}

This article has an editorial commentary: https://doi.org/10.1183/13993003.04423-2020

This article has supplementary material available from erj.ersjournals.com

Received: 30 Sept 2020 | Accepted: 13 Dec 2020

Copyright OERS 2021. This version is distributed under the terms of the Creative Commons Attribution NonCommercial Licence 4.0. 


\section{Introduction}

Severe acute respiratory syndrome coronavirus 2 (SARS-CoV-2) is the coronavirus that leads to coronavirus disease 2019 (COVID-19) and the current pandemic is the most critical ongoing global healthcare problem. To date, $>1.4$ million people have died of COVID-19 [1]. In Switzerland, as of November 25, 2020, a total of 309469 patients had been diagnosed with COVID-19 and 4030 patients had died [2].

COVID-19 is a heterogeneous disease with most patients experiencing mild illness and spontaneous recoveries, but a relevant subgroup of individuals require hospitalisation for pneumonia and other complications. In the initial reports from Wuhan, China, up to one-third of patients developed severe pneumonia with acute respiratory distress syndrome (ARDS) [3]. While we have already discovered much about the pathogenesis and treatment of the acute SARS-CoV-2 disease, intermediate and long-term outcomes are still unknown, particularly in survivors of severe disease courses.

Previous coronavirus infections include severe acute respiratory syndrome (SARS) and Middle East respiratory syndrome (MERS). Similar to COVID-19, SARS and MERS typically begin with an acute illness from which most patients recover after 2 weeks. However, up to one-third of SARS patients developed severe pulmonary complications and ARDS [4]. A subgroup of SARS survivors developed persistent lung parenchymal abnormalities, including pulmonary fibrosis $[5,6]$. The appearance of pulmonary fibrosis correlated with the severity and duration of the acute illness $[7,8]$, and radiological features of fibrosis persisted in $\sim 30 \%$ of patients after 3 and 6 months $[9,10]$. Older age and male sex were identified as risk factors for poor outcomes and development of lung fibrosis $[9,11]$.

With the anticipation of potential long-term sequelae after COVID-19, follow-up strategies have been proposed by several groups from the United States, United Kingdom, China and India [12-15].

Switzerland was one of the first European countries to be affected by COVID-19, with an increasing number of confirmed cases from February 25, 2020 onwards [2]. A nationwide lockdown was initiated on March 16, 2020 to prevent further spreading. 6 months later, we are facing not only challenges in acute COVID-19 patient care, but also in the follow-up and management of COVID-19 survivors presenting with sequelae of the disease.

We initiated the multicentre Swiss national COVID-19 lung study group to assess pulmonary sequelae of COVID-19. In this first analysis of our prospective cohort we report on chronic pulmonary sequelae of patients who had experienced mild to moderate (mild/moderate) and severe to critical (severe/critical) COVID-19, with the goal to improve the current understanding of the heterogeneous COVID-19 trajectories.

\section{Methods}

\section{Study setting, patients and clinical measurements}

This national, multicentre, prospective observational cohort study includes adults who survived acute COVID-19 and presented for clinical follow-up after either mild/moderate or severe/critical COVID-19.

Contributing centres for the Swiss COVID-19 lung study are the University Hospital Bern (Inselspital and Tiefenauspital), Lausanne University Hospital, University Hospital Geneva, University Hospital Zurich, Kantonspital St. Gallen, Kantonspital Freiburg, Hospital of Sion, Hospital of Basel (Claraspital), and Hospital of Tessin (Clinica Luganese Moncucco). All patients provided written informed consent before inclusion. Ethics approval was obtained prior to start of the study on May 1, 2020 (KEK 2020-00799).

Affiliations: 'Dept of Pulmonary Medicine, Inselspital, Bern University Hospital, University of Bern, Bern, Switzerland. ${ }^{2}$ Dept of Diagnostic, Interventional and Pediatric Radiology, Inselspital, Bern University Hospital, University of Bern, Bern, Switzerland. ${ }^{3}$ Radiodiagnostic and Interventional Radiology, CHUV-University Hospital, Lausanne, Switzerland. ${ }^{4}$ Service de Pneumologie, Hôpital du Valais, Sion, Switzerland. ${ }^{5}$ Lung Center, Kantonsspital St. Gallen, St. Gallen, Switzerland. ${ }^{6}$ Dept of Pneumology, University Hospital Zurich, Zurich, Switzerland. ${ }^{7}$ Clinic of Internal Medicine and Infectious Diseases, Clinica Luganese Moncucco, Lugano, Switzerland. ${ }^{8}$ Dept of Infectious Diseases, Bern University Hospital, University of Bern, Bern, Switzerland. ${ }^{9}$ Dept for BioMedical Research, University of Bern, Bern, Switzerland. ${ }^{10}$ Division of Pulmonary Medicine, Lausanne University Hospital (CHUV), University of Lausanne, Lausanne, Switzerland. ${ }^{11}$ Dept of Internal Medicine, University and Hospital of Fribourg, Fribourg, Switzerland. ${ }^{12}$ Dept of Pulmonary Medicine, Clinica Luganese Moncucco, Lugano, Switzerland. ${ }^{13}$ Dept of Pulmonary Medicine, St. Claraspital AG, Basel, Switzerland. ${ }^{14}$ Adult Intensive Care Unit, University Hospital and University of Lausanne, Lausanne, Switzerland. ${ }^{15}$ Dept of Intensive Care Medicine, Inselspital, Bern University Hospital, University of Bern, Bern, Switzerland. ${ }^{16}$ Division of Pulmonary Medicine, Geneva University Hospitals, Geneva, Switzerland.

Correspondence: Manuela Funke-Chambour, Division for Pulmonary Medicine, University Hospital Bern, Bern University, Bern 3010, Switzerland. E-mail: manuela.funke-chambourdinsel.ch 
Baseline information, e.g. symptoms at initial presentation, was retrieved from medical records. Pulmonary functional tests, measurement of diffusing capacity of the lung for carbon monoxide $\left(D_{\mathrm{LCO}}\right)$ and 6-min walk tests (6MWT) were performed using established protocols [16-19]. Respiratory muscle strength was estimated by measurement of maximum static inspiratory pressure and maximum static expiratory pressure at the mouth [20]. Chest computed tomography (CT) scans were performed in clinically symptomatic patients.

Patients were stratified into the following two groups according to four severity grades described by the World Health Organization: 1) mild disease, or moderate disease with clinical signs of pneumonia and peripheral oxygen saturation $\left(S_{\mathrm{pO}_{2}}\right) \geqslant 90 \%$ (mild/moderate); 2) severe disease with pneumonia and $S_{\mathrm{pO}_{2}}$ $<90 \%$, respiratory rate $>30$ breaths. $\mathrm{min}^{-1}$ or critical disease, i.e. ARDS, sepsis, septic shock and multiorgan failure (severe/critical) [21].

\section{Chest CT acquisition}

Standard chest CT scans were acquired according to the local protocols in participating centres. 52 follow-up chest CT scans from the 113 included patients were available. All chest CT scans were reconstructed with $\sim 1 \mathrm{~mm}$ slice thickness. Application of iodine contrast agents was only performed if pulmonary embolism was suspected and/or in case of clinical deterioration. Multiplane reconstructions were performed in axial, coronal and sagittal planes as required. All images were reconstructed with lung and soft tissue kernels and stored in the local picture archiving and communication system (PACS).

\section{Image analysis}

All available CT scans were collected from Bern and Lausanne and de-identified in the local image archive system (PACS Carestream Health, Rochester, NY, USA for Lausanne; Sectra PACS, IDS7, Linköping, Sweden for Bern). Two subspecialised chest radiologists from two tertiary-care centres performed a consensus readout, blinded to the clinical status of the patients. For the reading process, the radiologists reviewed the cases online via screen sharing (Cisco Webex, Milpitas, CA, USA), as the current pandemic regulations restrict physical meetings. The readers assessed the presence of the following chest CT patterns: consolidation, ground-glass opacities (focal, multifocal, diffuse), mosaic attenuation pattern (hypoattenuating areas, hyperattenuating areas), perilobular consolidation (organising pneumonia-like pattern), reticulations, architectural distortion, honeycombing, traction bronchiectasis, pneumatoceles, curvilinear lines, nodules, pleural thickening or pleural effusion, mucus plugging, vascular abnormalities and additional findings were annotated separately. Additionally, pattern distribution was recorded (upper lobe, middle lobe/lingual, lower lobe). A semiquantitative estimation of the disease extent was performed, based on the system proposed by Francone et al. [22]. The CT score is derived from extent of lobar involvement based on a five-point scale $(0: 0 \%, 1:<5 \%, 2: 5-25 \%, 3: 26-50 \%, 4: 51-75 \%, 5:>75 \%$; range 0-5; global score 0-25).

\section{Statistical analysis}

Descriptive statistics are reported as mean \pm SD or median (interquartile range (IQR)). Differences between mild/moderate and severe/critical COVID-19 groups were analysed for statistical significance by Chi-squared or Fisher's exact test for categorical variables and by two-sample t-test or Wilcoxon rank-sum test for continuous variables as applicable. The associations of demographic factors, pulmonary and physical function tests, and radiological signs with the COVID-19 severity groups were estimated using linear or logistic regression models where applicable. Models were adjusted for potential confounders with either conceptual importance (age, sex) or a statistically significant relationship to COVID-19 severity $(\mathrm{p}<0.1)$. Model fit was examined using the area under the receiver operating characteristic curve (AUC). 95\% confidence intervals for AUC were calculated from bootstrap resampling with 2000 repetitions. The final models were selected based on overall AUC. A two-sided $\mathrm{p}<0.05$ was considered for all comparisons. Data were analysed using R (version 3.6.0; R Foundation for Statistical Computing, Vienna, Austria).

\section{Results}

\section{Baseline characteristics and symptoms}

Collaborating centres included 113 patients ( $\mathrm{n}=66$ after severe/critical and $\mathrm{n}=47$ after mild/moderate COVID-19) from May 1, 2020 until September 15, 2020 (figure 1). Median (IQR) time from initial symptoms to the follow-up visit was 128 (108-144) days.

Patients who survived severe/critical COVID-19 were older than patients followed-up after mild/moderate disease (mean \pm SD age $60.3 \pm 12$ versus $52.9 \pm 11$ years, with equal sex distribution) (table 1 ). Smoking history in mild/moderate and severe/critical COVID-19 patients was not significantly different. However, mean \pm SD body mass index (BMI) was significantly higher in patients with severe/critical disease than in those with $\mathrm{mild} /$ moderate disease $\left(29.8 \pm 5.7\right.$ versus $\left.25.5 \pm 4.7 \mathrm{~kg} \cdot \mathrm{m}^{-2}, \mathrm{p}=0.02\right)$. Initial comorbidities in both groups are 


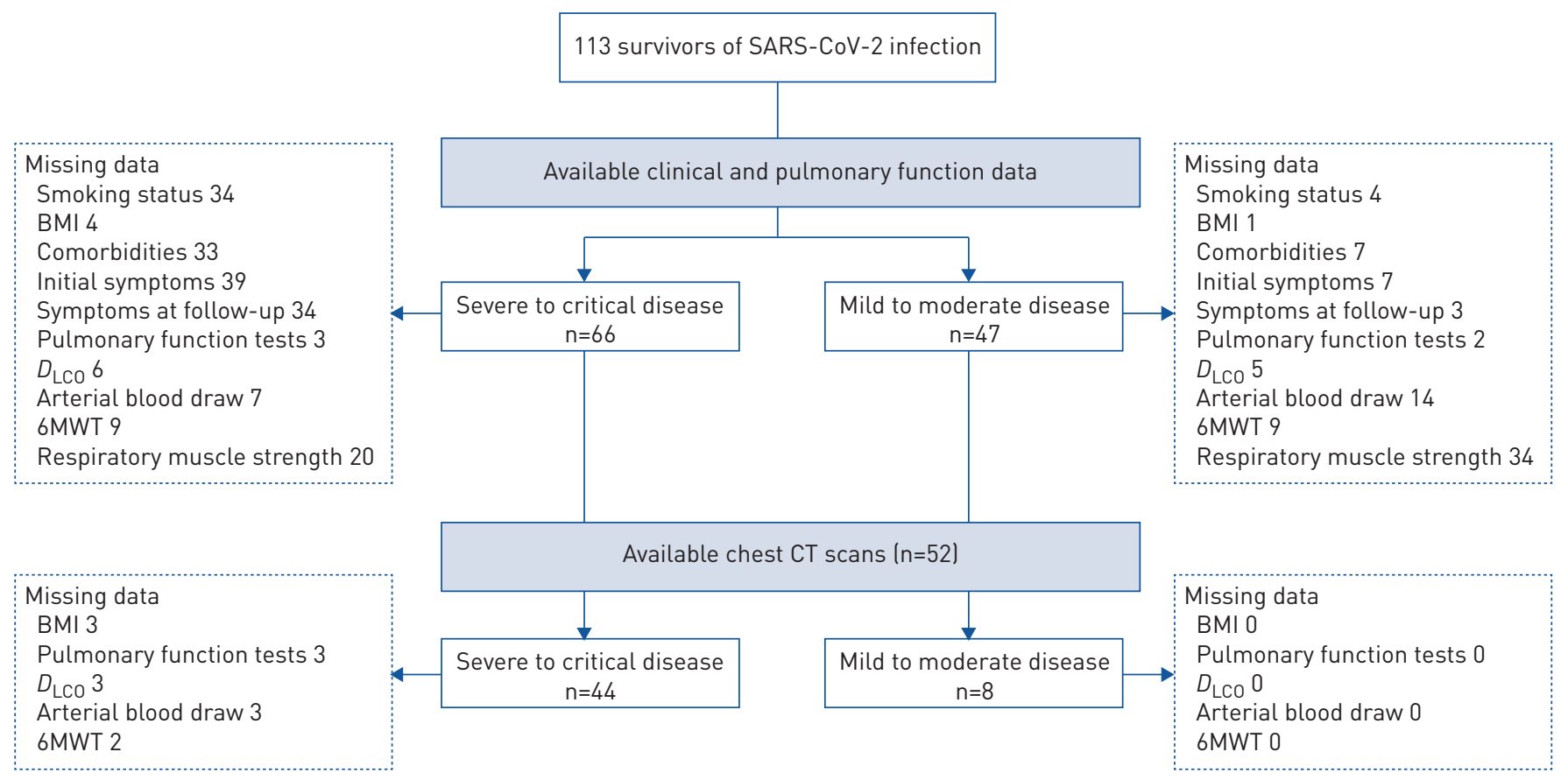

FIGURE 1 Preferred Reporting Items for Systematic Reviews and Meta-Analyses flow diagram of the study. SARS-CoV-2: severe acute respiratory syndrome coronavirus 2; BMI: body mass index; $D_{\mathrm{LCO}}$ : diffusing capacity of the lung for carbon monoxide; 6MWT: 6-min walk test; CT: computed tomography.

listed in table 1. At follow-up, patients in both COVID-19 severity groups did not indicate relevant cough (median (IQR) cough visual analogue scale $0(0-2)$ ). However, patients reported persistent exertional dyspnoea in both groups (median (IQR) modified Medical Research Council score $1(0-1)$ ) at follow-up.

A sensitivity analysis excluding patients with previously diagnosed chronic lung diseases was performed to estimate if the observed differenced in follow-up pulmonary function were mainly driven by pre-existing lung diseases. Supplementary table S1 demonstrates the mainly unchanged findings in this subgroup.

\section{Pulmonary function, physical performance and oxygenation}

Overall, average pulmonary function was normal in patients after mild/moderate COVID-19. Patients after severe/critical COVID-19 had generally lower lung volumes that were still within the normal range, whereas average measures of diffusion capacity $\left(D_{\mathrm{LCO}}\right)$, physical performance and oxygenation were reduced. Specifically, total lung capacity (TLC), forced vital capacity (FVC), forced expiratory volume in $1 \mathrm{~s}\left(\mathrm{FEV}_{1}\right)$ and $D_{\mathrm{LCO}}$ were significantly lower in patients after severe/critical COVID-19 compared to patients after mild/moderate disease. Furthermore, COVID-19 patients with mild/moderate disease course had a lower ratio of $\mathrm{FEV}_{1} / \mathrm{FVC}$ than those with severe/critical illness (table 1). Patients with severe/critical disease had impaired and significantly lower $D_{\text {LCO }}$ compared to patients with mild/moderate disease (mean \pm SD $73.2 \pm 18.4 \%$ pred versus $95.3 \pm 20.3 \%$ pred, $\mathrm{p}=0.003$ ). 6 -min walk distance (6MWD) was $120 \mathrm{~m}$ lower in the severe/critical disease group, with an average $S_{\mathrm{pO}_{2}}$ decrease of $5.6 \pm 3.8 \%$ in the severe/critical group compared to $2.6 \pm 3.1 \%$ in the mild/moderate disease group $(\mathrm{p}=0.02)$. Similarly, 4 months after COVID-19, former severe/critical patients had lower arterial oxygen tension $\left(P_{\mathrm{aO}_{2}}\right)$ compared to former mild/moderate patients $\left(P_{\mathrm{aO}_{2}} 79 \pm 12.2\right.$ versus $\left.87.5 \pm 9 \mathrm{mmHg}, \mathrm{p}=0.0002\right)$ (table 1). Respiratory muscle strength did not differ in both groups.

Within the subgroup of patients who needed mechanical ventilation (39 patients with severe/critical disease) several measures of pulmonary function at follow-up were negatively correlated with the duration of mechanical ventilation during acute COVID-19 (supplementary table S2). Specifically, $D_{\text {LCO }}$ and TLC showed a moderately strong negative correlation with duration of ventilation $(\mathrm{r}=-0.43, \mathrm{p}=0.008$ and $\mathrm{r}=-0.42$, $\mathrm{p}=0.01$, respectively).

Clinical associations with a severe/critical disease course

Unadjusted analysis showed patients after severe/critical COVID-19 being 7.4 (95\% CI 3-11.7) years older $(\mathrm{p}=0.002)$ and having a $4.4(95 \%$ CI $2.3-6.4) \mathrm{kg} \cdot \mathrm{m}^{-2}$ higher BMI $(\mathrm{p}<0.001)$ than patients after mild/ moderate disease. FVC \% predicted was 9\% (95\% CI 1.5-16.4\%) lower and TLC \% predicted was $16 \%$ 
TABLE 1 Baseline characteristics and follow-up findings in patients with severe/critical and mild /moderate coronavirus disease 2019

\begin{tabular}{|c|c|c|c|}
\hline & Severe/critical disease & Mild/moderate disease & p-value ${ }^{\#}$ \\
\hline Patients n & 66 & 47 & \\
\hline \multicolumn{4}{|l|}{ Baseline characteristics } \\
\hline Male/female $\mathrm{n} / \mathrm{n}$ & $40 / 26$ & $27 / 20$ & 0.89 \\
\hline Age years & $60.3 \pm 12.0$ & $52.9 \pm 10.9$ & $<0.001$ \\
\hline Ever-smokers $\%$ & 56 & 37 & 0.16 \\
\hline $\mathrm{BMI} \mathrm{kg} \cdot \mathrm{m}^{-2}$ & $29.8 \pm 5.7$ & $25.5 \pm 4.7$ & 0.02 \\
\hline $\mathrm{D}-\mathrm{dimer}^{+} \mu \mathrm{g} \cdot \mathrm{L}^{-1}$ & $1011(366-1989)$ & $387(1-658)$ & 0.26 \\
\hline Mechanical ventilation $\S \%$ & 71 & & \\
\hline Duration of mechanical ventilation $\S$ days & $11.9(9.5-18)$ & & \\
\hline \multicolumn{4}{|l|}{ Comorbidities $\%$} \\
\hline Interstitial lung disease & 6 & 3 & 0.58 \\
\hline COPD & 12 & 3 & 0.18 \\
\hline Asthma & 9 & 19 & 0.32 \\
\hline Arterial hypertension & 55 & 8 & 0.003 \\
\hline Diabetes & 35 & 0 & 0.04 \\
\hline GORD & 10 & 9 & 1.00 \\
\hline Sleep apnoea & 16 & 3 & 0.09 \\
\hline Chronic heart failure & 10 & 9 & 1.00 \\
\hline Chronic renal failure & 19 & 0 & 0.009 \\
\hline Cancer & 6 & 5 & 1.00 \\
\hline Depression or anxiety & 12 & 7 & 0.66 \\
\hline \multicolumn{4}{|l|}{ Pulmonary function at follow-up ${ }^{f}$} \\
\hline $\mathrm{FEV}_{1} / \mathrm{FVC} \%$ & $94.7 \pm 13.7$ & $84.2 \pm 14.3$ & $<0.001$ \\
\hline TLC L & $5.22 \pm 1.5$ & $6.5 \pm 1.6$ & 0.050 \\
\hline TLC \% pred & $86.0 \pm 20.0$ & $102.0 \pm 19.3$ & 0.047 \\
\hline FVC L & $3.28 \pm 1.01$ & $4.12 \pm 1.2$ & $<0.001$ \\
\hline FVC $\%$ pred & $86.6 \pm 20.1$ & $95.6 \pm 17.9$ & 0.02 \\
\hline $\mathrm{FEV}_{1} \mathrm{~L}$ & $2.64 \pm 0.8$ & $3.34 \pm 1.1$ & $<0.001$ \\
\hline $\mathrm{FEV}_{1} \%$ pred & $89.4 \pm 20.7$ & $94.0 \pm 15.6$ & 0.19 \\
\hline$D_{\text {Lco }} \%$ pred & $73.2 \pm 18.4$ & $95.3 \pm 20.6$ & 0.003 \\
\hline$P_{\text {Imax }} \mathrm{kPa}$ & $10.3 \pm 8.8$ & $8.1 \pm 2.6$ & 0.14 \\
\hline$P_{\text {Emax }} \mathrm{kPa}$ & $8.7 \pm 3.3$ & $10.3 \pm 4.1$ & 0.20 \\
\hline \multicolumn{4}{|l|}{ Oxygenation at follow-up $f$} \\
\hline$P_{\mathrm{aO}_{2}} \mathrm{mmHg}$ & $79.0 \pm 12.2$ & $87.5 \pm 9.0$ & 0.0002 \\
\hline $6 \mathrm{MWD} \mathrm{m}$ & $456 \pm 105$ & $576 \pm 78$ & 0.001 \\
\hline $\mathrm{O}_{2}$ nadir on $6 \mathrm{MWT}$ & $90 \pm 4.5$ & $93 \pm 3.1$ & 0.001 \\
\hline $\mathrm{O}_{2}$ desaturation on $6 \mathrm{MWT}$ & $5.6 \pm 3.8$ & $2.6 \pm 3.1$ & 0.02 \\
\hline
\end{tabular}

Data are presented as mean \pm SD or median (interquartile range), unless otherwise stated. BMI: body mass index; GORD: gastro-oesophageal reflux disease; $\mathrm{FEV}_{1}$ : forced expiratory volume in $1 \mathrm{~s}$; FVC: forced vital capacity; TLC: total lung capacity; $D_{\mathrm{LCO}}$ : diffusing capacity of the lung for carbon monoxide; $P_{\text {Imax }}$ : maximal inspiratory pressure; $P_{\text {Emax }}$ : maximal expiratory pressure; $P_{\mathrm{aO}_{2}}$ : arterial oxygen tension; 6MWD: 6-min walk distance; 6MWT: 6-min walk test. \#: p-values from two-sample t-tests, Wilcoxon rank sum test, Chi-squared test or Fisher's exact test; ${ }^{\text {ๆ }}: \mathrm{n}=38$ missing; ${ }^{+}: \mathrm{n}=59$ missing; ${ }^{\S}: \mathrm{n}=11$ missing; ${ }^{f}: \mathrm{n}=72$.

(95\% CI 8.3-23.6\%) lower after severe/critical COVID-19 than after mild/moderate COVID-19. After adjustment for age, sex and BMI, TLC remained significantly lower in the severe/critical disease group (14.6\% pred, 95\% CI 6.3-22.8\% pred; $\mathrm{p}<0.001$ ), whereas FVC lost statistical significance (5.6\% pred, $95 \%$ CI $-2.6-13.8 \%$ pred; $\mathrm{p}=0.18)$. $D_{\text {LCO }}$ was significantly higher after mild/moderate COVID-19 on unadjusted analysis (22.1\% pred, 95\% CI 14.4-29.8\% pred; $\mathrm{p}=0.001$ ), and after adjustment for the aforementioned confounders (20.9\% pred, 95\% CI $12.4-29.4 \%$ pred; $\mathrm{p}=0.01$ ).

Similarly, survivors of severe/critical COVID-19 had a markedly lower 6MWD on unadjusted (120 m, 95\% CI 80-160 m; p=0.001) and adjusted analyses ( $86 \mathrm{~m}, 95 \%$ CI 45-127 m; p=0.001). After adjustment for age, sex and BMI, minimal $S_{\mathrm{pO}_{2}}$ on $6 \mathrm{MWD}$ was $2.2 \%$ (95\% CI $\left.0.4-4.0 \%, \mathrm{p}=0.01\right)$ and $P_{\mathrm{aO}_{2}}$ was $5 \mathrm{mmHg}$ (95\% CI $0.3-10.2 \mathrm{mmHg})$ lower $(\mathrm{p}=0.06)$ after severe/critical disease.

Figure 2 shows the association of follow-up clinical variables with initial severe/critical and mild/moderate COVID-19. Table 2 illustrates the final multivariable model including clinical and functional variables 


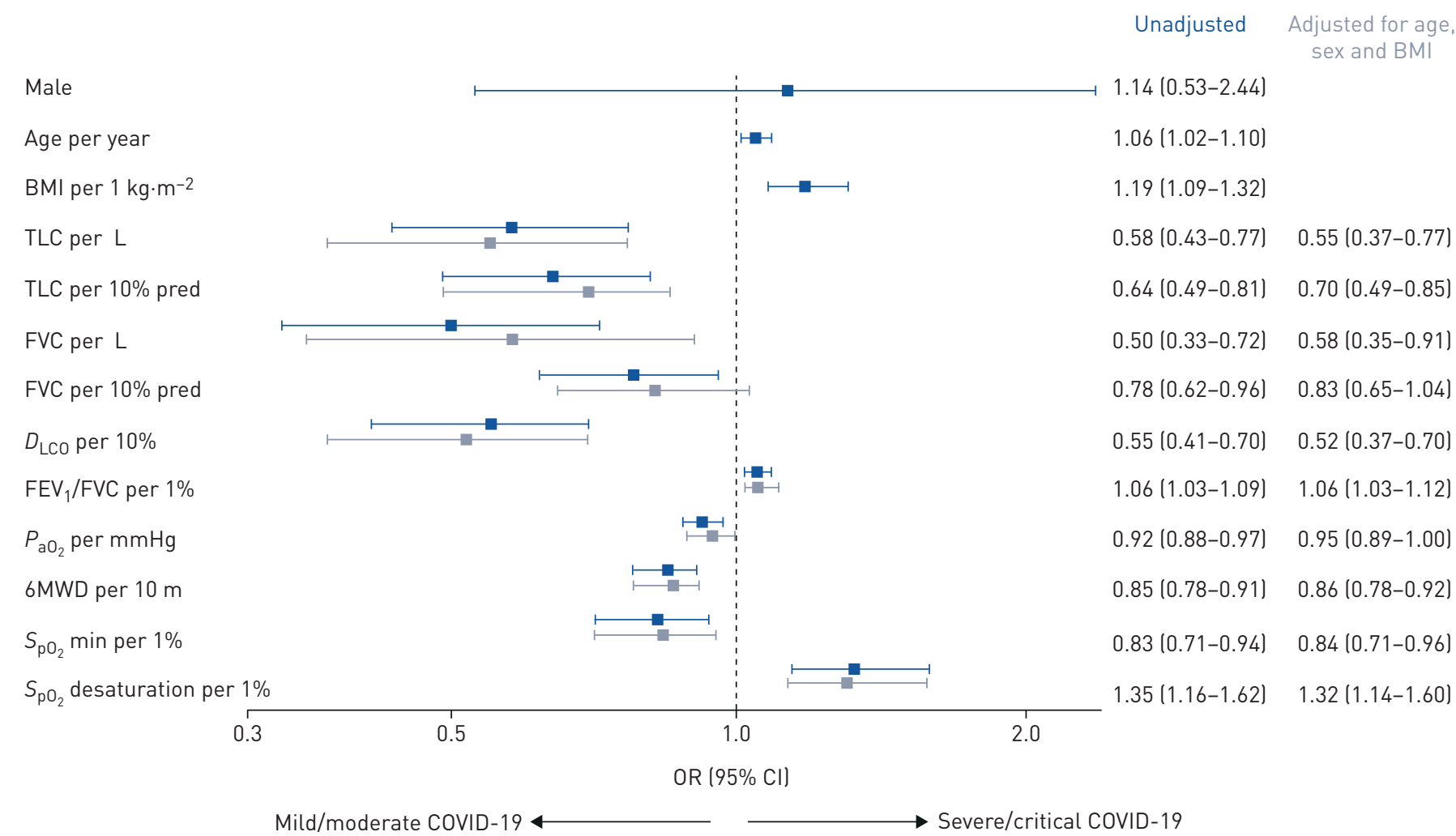

FIGURE 2 Variables associated with past coronavirus disease 2019 (COVID-19) severity. Association of demographic and functional parameters with mild/moderate and severe/critical COVID-19. Odds ratios and corresponding $95 \%$ confidence intervals from unadjusted analysis and individual multivariable models for each parameter adjusting for confounding by age and sex. BMI: body mass index; TLC: total lung capacity; FVC: forced vital capacity; $D_{\mathrm{LCO}}$ : diffusing capacity of the lung for carbon monoxide; $\mathrm{FEV}_{1}$ : forced expiratory volume in $1 \mathrm{~s} ; P_{\mathrm{aO}_{2}}$ : arterial oxygen tension; 6MWD: 6-min walk distance; $\mathrm{S}_{\mathrm{pO}_{2}}$ : peripheral oxygen saturation.

statistically and conceptually associated with a severe/critical course of COVID-19 in our cohort. High $D_{\text {LCO }} \%$ predicted at follow-up was the factor with the strongest independent association with a more favourable previous course of disease $(\mathrm{p}=0.01)$. A good model fit was demonstrated by the overall AUC of 0.95 (95\% CI $0.88-1.00)$.

\section{Radiological features}

In our cohort, typical radiological follow-up sequelae of COVID-19 included uni- or multi-lobular hypoattenuated areas without or with bulging of the lobular margins, ground-glass opacities with a mosaic attenuation pattern, linear/curvilinear densities, reticulations, honeycombing, traction bronchiectasis with

TABLE 2 Complete clinical multivariable model for severe/critical disease

\begin{tabular}{|c|c|c|}
\hline & OR $(95 \% \mathrm{CI})$ & $\mathrm{p}$-value \\
\hline$D_{\text {Lco }}$ per $10 \%$ pred & $0.59(0.37-0.87)$ & 0.01 \\
\hline Age per year & $0.98(0.92-1.05)$ & 0.62 \\
\hline Male & $1.98(0.50-8.56)$ & 0.34 \\
\hline BMI per $1 \mathrm{~kg} \cdot \mathrm{m}^{-2}$ & $1.19(1.03-1.41)$ & 0.02 \\
\hline 6MWD per $10 \mathrm{~m}$ & $0.88(0.67-0.95)$ & 0.01 \\
\hline Minimum $S_{\mathrm{po}_{2}}$ per $1 \%$ & $0.80(0.44-0.95)$ & 0.07 \\
\hline
\end{tabular}

Effect estimates indicate the associations of the variables with severe/critical disease in the context of the complete multivariable model, e.g. for every 10 -m increase in 6-min walk distance (6MWD), the odds for severe/critical disease decreases by $12 \%$ adjusting for age, sex, body mass index (BMI), diffusing capacity of the lung for carbon monoxide $\left(D_{\mathrm{LCO}}\right)$ and minimal oxygen saturation on 6-min walk test $\left(\mathrm{S}_{\mathrm{pO}_{2}}\right)$. The overall area under the receiver operating curve of the multivariable model including $D_{\mathrm{LCO}}$, age, sex, BMI, 6MWD and minimal $\mathrm{S}_{\mathrm{pO}_{2}}$ was $0.95(95 \% \mathrm{Cl} 0.88-1.00)$. 
architectural distortion in various locations, as well as pneumatoceles (figure 3). Extensive pulmonary fibrosis was rarely observed (supplementary figure S1).

Radiological features that were significantly more prevalent after severe/critical than after mild/moderate COVID-19 included mosaic attenuation pattern with hypoattenuated areas (66\% versus 13\%, $\mathrm{p}=0.007)$, and reticulations $(59 \%$ versus $13 \%, \mathrm{p}=0.02)$ and architectural distortion $(52 \%$ versus $13 \%, \mathrm{p}=0.055)$ were marginally more frequent in severe/critical disease (supplementary table S2).

Our patients with a mosaic attenuation pattern with hypoattenuation areas on chest CT scan were more than 13 times more likely to have suffered a severe/critical disease course in our study (table 3). This association remained statistically significant with accounting for potential confounding by age and sex (OR 13, 95\% CI 1.7-239 $\mathrm{p}=0.03)$. Similarly, reticulations increased the odds of past severe/critical COVID-19 10-fold (95\% CI 1.6-198, p=0.04) (table 3).

\section{Discussion}

To our knowledge, this is the first European study reporting on respiratory follow-up outcomes after SARS-CoV-2 infection. After an average observation time of 4 months, our cohort reveals impairments in pulmonary function and physical performance that were more pronounced in patients with previously

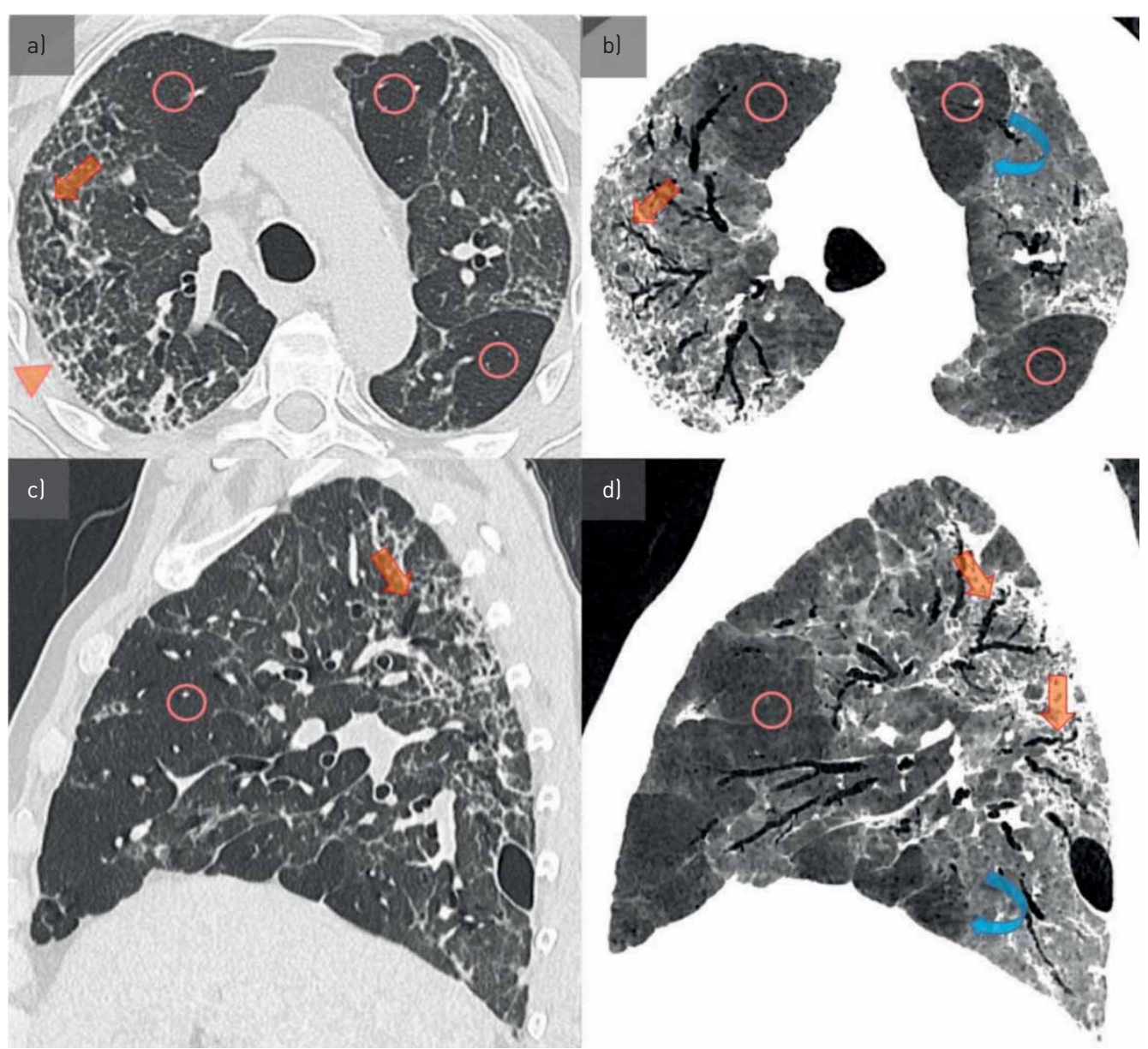

FIGURE 3 Characteristic radiological changes of a patient with severe sequelae 3 months after coronavirus disease 2019 (COVID-19) pneumonia. Extensive involvement of both lungs is present in a patient 3 months after severe COVID-19 pneumonia. Diffuse mosaic attenuation pattern in all lung lobes seen on a) axial 1-mm-thick computed tomography (CT) scan and b) 10-mm-thick minimum intensity projection (mIP) slices, c) 1-mm-thick CT and d) 10-mm-thick mIP sagittal reformats in lung windowing. This combines classical features of lung fibrosis with architectural distortion, reticulations, honeycombing (arrowhead in a) and traction bronchiectasis (straight arrows in a-d), as well as sharply demarcated areas of low attenuation in both lungs (circles in $\mathrm{a}-\mathrm{d}$ ). Clusters of contiguous hypoattenuating lobules and traction bronchiectasis are better visualised on mIP images with narrow window settings ( $b$ and $d$ ). Note the bulging of the interlobular septae ( $b$ and $d$, curved arrows) as well as the subpleural pneumatocele in $c$ and $d$. 
TABLE 3 Unadjusted and adjusted association of radiological features with previous severe/ critical coronavirus disease 2019

\begin{tabular}{|c|c|c|c|c|}
\hline & \multicolumn{2}{|c|}{ Unadjusted } & \multicolumn{2}{|c|}{ Adjusted for age and sex } \\
\hline & OR $(95 \% \mathrm{CI})$ & p-value ${ }^{\#}$ & OR $(95 \% \mathrm{CI})$ & p-value ${ }^{\#}$ \\
\hline Hypoattenuation & $13.5(2.1-265)$ & 0.02 & $11.7(1.7-239)$ & 0.03 \\
\hline Reticulations & $10.1(1.6-198)$ & 0.04 & $8.73(1.3-174)$ & 0.06 \\
\hline
\end{tabular}

Odds ratios indicate the associations of the radiological variables at follow-up with severe/critical disease, e.g. the odds of severe/critical disease is 11.7 times higher for a patient with than for a patient without hypoattenuation, adjusting for age and sex. ${ }^{\#}$ : p-value for the radiological sign.

severe/critical COVID-19 courses, compared to those with mild/moderate illness. Specifically, $D_{\text {LCO }} \%$ predicted at 4 months was the most important independent correlate of a more severe initial disease. Furthermore, in the subcohort of patients with available chest CT scan, we identified a mosaic attenuation pattern with hypoattenuated areas of various size limited by geographic margins with bulging of secondary pulmonary lobules, as well as reticulations as distinct radiological features after severe/critical COVID-19.

Information on risk factors for the development of COVID-19 pneumonia and a severe disease course is increasing, and our study supports many of the findings from previous reports [23-25]. In our cohort, we confirm that age and age-associated comorbidities significantly contribute to the inter-individual heterogeneity in the severity of acute COVID-19 [24]. For example, with obesity emerging as a risk factor for severe COVID-19 and mortality [26-28], findings from the Swiss COVID-19 lung cohort confirm the importance of this modifiable risk factor.

COVID-19 primarily affects the lung and airways and may lead to respiratory failure [25]. 5-year survivors of ARDS were found to be functionally impaired with a median 6MWD 76\% of predicted [29]. Similarly, a meta-analysis on long-term outcomes after SARS and MERS identified a reduced 6MWD and $D_{\text {LCO }}$ compared to healthy individuals [30]. A 3-month follow-up study of a Wuhan cohort (mostly mild pneumonia) demonstrated reduced pulmonary function in 14 out of 55 cases, including nine out of 55 cases with reduced $D_{\mathrm{LCO}}$ [31]. In our study, reduced $D_{\mathrm{LCO}}$, decreased $6 \mathrm{MWD}$ and desaturation during 6MWT were associated with the severely impaired COVID-19 phenotype, and importantly this relationship was not confounded by age, sex or BMI.

The prevalence and extent of pulmonary function and physical impairment after different clinical courses of COVID-19 are still uncertain. In our cohort we demonstrate lower lung volumes (TLC, FVC and FEV F $_{1}$ in patients after severe/critical COVID-19; the higher $\mathrm{FEV}_{1} / \mathrm{FVC}$ ratio in the severe/critical subgroup suggests a tendency toward a restrictive physiology, and the lack of difference in respiratory muscle strength suggests a lung parenchymal issue rather than a respiratory muscle issue. Furthermore, we demonstrate a negative correlation between the duration of mechanical ventilation during the acute disease and pulmonary function at 4-month follow-up. This might be due to a prolonged impairment after very severe COVID-19 or related to more severe disease course in susceptible patients. Alternatively, ventilator induced lung-injury is a well-described challenge post-ARDS, which can impact on pulmonary function after recovery from the acute illness [29].

On chest CT scans, acute COVID-19 typically presents with progressive ground-glass opacities, alveolar consolidations with a common subpleural and basal location, rounded lesions, crazy-paving pattern, linear densities, parenchymal bands and architectural distortion perhaps representing organising pneumonia and/or fibrotic changes [32]. This presents as an overall radiological picture that is distinct from other viral infections, such as influenza $[33,34]$. Similar to the clinical evolution, the trajectory of radiological patterns is reported to be heterogeneous. After a mean observation time of 10 days, lung parenchymal abnormalities seem to have improved in most cases, while some patients persistently show mild signs of pulmonary fibrosis [35]. At short-term follow-up, the most frequent radiological abnormalities included ground-glass opacities, consolidation and parenchymal bands [36]. Reports on medium- or long-term development of radiological abnormalities are currently very limited.

4 months after the initial diagnosis the predominant finding in our severe/critical subcohort was a mosaic attenuation pattern, characterised by abnormally hypodense areas corresponding to one or several contiguous secondary pulmonary lobules alternating with normal or abnormally hyperdense areas (i.e. ground-glass attenuation), as well as more frequent reticulations. In most cases, there was a sharp demarcation and delineation of the margins of the involved secondary pulmonary lobules, with sometimes hyperdense bands silhouetting the abnormal lobules. The combination of mosaic attenuation pattern and 
impaired $D_{\text {LCO }}$ can either be attributed to abnormalities in the distal airways such as constrictive bronchiolitis with air trapping and secondary reflex vasoconstriction or a primary pulmonary vascular disease that may induce secondary airway disease. Both mechanisms can cause ventilation/perfusion mismatch contributing to the reduced physical performance and hypoxaemia that we observed in our severe/critical subcohort. Endothelial injury and alveolar capillary microthrombosis have been discussed as underlying mechanisms of pulmonary vascular disease [37]. In addition, airway disease and air trapping have been described after adult ARDS in the setting of influenza, MERS and SARS [6, 38, 39]. Overall, observations from previous studies combined with the high prevalence of the mosaic attenuation pattern specifically in our severe/critical COVID-19 subcohort suggest that this pattern is an important late feature of severe COVID-19. Signs of fibrosis that we observed in our cohort (reticulations, bronchiectasis and honeycombing) are frequently encountered in survivors of ARDS. While initial observations reported fibrosis after COVID-19-associated ARDS [38], mosaic attenuation and air trapping have not been described after COVID-19 before. We recently reported that radiological mosaic attenuation and air trapping might indicate small airway disease [40]. This finding has been reported previously after coronavirus infection and virus-associated $\operatorname{ARDS}[6,38,39]$. The radiological and functional abnormalities observed in our study might represent residual damage after ARDS in general, after ventilator-induced lung injury, or specifically after SARS-CoV-2 infection. Potential development of progressive interstitial lung disease after COVID-19 might be attributed to stimulation of autoimmune pathways triggered by SARS-CoV-2 or progression from pre-existing interstitial lung abnormalities to clinically significant interstitial lung disease.

Prolonged functional impairment with slow recovery over several years after ARDS is not uncommon [29]; however, potential long-term consequences specifically after COVID-19 still need to be investigated in future observational studies.

The design of our study does not allow any inference of the prevalence of severe/impaired and mild/ moderate COVID-19, as patients who died were not included, and we did not include asymptomatic patients for follow-up. Initial pulmonary function tests and imaging were not available; consequently, patients who experienced severe/critical disease might have had a predisposing undiagnosed lung disease and poorer pulmonary function at follow-up. Patients were questioned thoroughly for symptoms and a medical history that might have indicated previous lung disease. However, asymptomatic underlying chronic lung disease cannot be completely excluded. In addition, initial interstitial lung abnormalities or involvement of the airways in COVID-19 infection cannot be assessed due to the lack of previous chest CT scan in most cases. However, our findings combined with previous studies support the hypothesis that severe COVID-19 causes a medium-term decrease in $D_{\mathrm{LCO}}$, limitations in gas exchange and a spectrum of radiological features not previously reported, pointing towards a considerable small-airway component.

In conclusion, with the analysis of pulmonary function, physical performance, oxygenation and radiological findings 4 months after COVID-19, our study adds to the growing body of evidence on post-COVID-19 trajectories. We identified $D_{\text {LCO }} \%$ predicted at 4 months as the single most important factor associated with severe/critical respiratory COVID-19, which translates to reduced walking distance and oxygen desaturation on exercise. The peculiar radiological presentation should be further investigated to provide an overall assessment of the disease in conjunction with other functional parameters. These results emphasise the importance of a systematic follow-up after severe and critical COVID-19, with appropriate management of pulmonary sequelae.

Acknowledgements: We would like to thank all participants in this study, our study coordinator Kurt De Jaegere, all study nurses and all other staff who helped to conduct this study: Anja Renner (Bern), Jasmin Lippuner (Bern), Ilena Müller (Bern), Wiasemsky Mary-Line (Lausanne), Estelle Clément (Lausanne), Noriane Sievi (Zürich), Verica Filipin (St. Gallen), Sandra Widmer (St. Gallen), Kei Kozu (St. Gallen), Florent Baty (St. Gallen), Pier Andrea Maida (Lugano), Maira Biggiogero (Lugano), Marlène Salamin (Sion), Martine Rime (Fribourg), and Sandrine Foucras (Fribourg).

Data are available upon request and after approval of the steering committee of the Swiss COVID-19 lung study group.

Conflict of interest: S.A. Guler has nothing to disclose. L. Ebner has nothing to disclose. C. Beigelman reports personal fees for lectures from AstraZeneca and Boehringer, outside the submitted work. P-O. Bridevaux has nothing to disclose. M. Brutsche has nothing to disclose. C. Clarenbach reports personal fees from Roche, Novartis, Boehringer, GSK, AstraZeneca, Sanofi, Vifor and Mundipharma, outside the submitted work. C. Garzoni has nothing to disclose. T.K. Geiser has nothing to disclose. A. Lenoir has nothing to disclose. M. Mancinetti has nothing to disclose. B. Naccini has nothing to disclose. S.R. Ott has nothing to disclose. L. Piquilloud has nothing to disclose. M. Prella has nothing to disclose. Y-A. Que has nothing to disclose. P.M. Soccal has nothing to disclose. C. von Garnier has nothing to disclose. M. Funke-Chambour has nothing to disclose.

Support statement: Lungenliga Bern, Johanna Dürmüller Foundation, Bern Center for Precision Medicine (BCPM) and the Department für Lehre und Forschung (DLF) of the Inselspital Bern funded parts of this study but had no role in the conduct of the investigation. Funding information for this article has been deposited with the Crossref Funder Registry. 


\section{References}

1 World Health Organization (WHO). WHO Coronavirus Disease (COVID-19) Dashboard. covid19.who.int/ Date last accessed: November 25, 2020. Date last updated: November 25, 2020.

2 Bundesamt für Gesundheit (BAG). Coronavirus: Situation in Switzerland. www.bag.admin.ch/bag/de/home/ krankheiten/ausbrueche-epidemien-pandemien/aktuelle-ausbrueche-epidemien/novel-cov/situation-schweiz-undinternational.html Date last accessed: November 25, 2020. Date last updated: November 25, 2020.

3 Huang C, Wang Y, Li X, et al. Clinical features of patients infected with 2019 novel coronavirus in Wuhan, China. Lancet 2020; 395: 497-506.

4 Tsui PT, Kwok ML, Yuen H, et al. Severe acute respiratory syndrome: clinical outcome and prognostic correlates. Emerg Infect Dis 2003; 9: 1064-1069.

5 Cheung OY, Chan JW, Ng CK, et al. The spectrum of pathological changes in severe acute respiratory syndrome (SARS). Histopathology 2004; 45: 119-124.

6 Ketai L, Paul NS, Wong KT. Radiology of severe acute respiratory syndrome (SARS): the emerging pathologic-radiologic correlates of an emerging disease. J Thorac Imaging 2006; 21: 276-283.

7 Tse GM, To KF, Chan PK, et al. Pulmonary pathological features in coronavirus associated severe acute respiratory syndrome (SARS). J Clin Pathol 2004; 57: 260-265.

8 Hwang DM, Chamberlain DW, Poutanen SM, et al. Pulmonary pathology of severe acute respiratory syndrome in Toronto. Mod Pathol 2005; 18: 1-10.

9 Hui DS, Wong KT, Ko FW, et al. The 1-year impact of severe acute respiratory syndrome on pulmonary function, exercise capacity, and quality of life in a cohort of survivors. Chest 2005; 128: 2247-2261.

10 Ngai JC, Ko FW, Ng SS, et al. The long-term impact of severe acute respiratory syndrome on pulmonary function, exercise capacity and health status. Respirology 2010; 15: 543-550.

11 de Wit E, van Doremalen N, Falzarano D, et al. SARS and MERS: recent insights into emerging coronaviruses. Nat Rev Microbiol 2016; 14: 523-534.

12 Zheng Z, Yao Z, Wu K, et al. Patient follow-up after discharge after COVID-19 pneumonia: considerations for infectious control. J Med Virol 2020; 92: 2412-2419.

13 Balachandar V, Mahalaxmi I, Subramaniam M, et al. Follow-up studies in COVID-19 recovered patients - is it mandatory? Sci Total Environ 2020; 729: 139021.

14 Raghu G, Wilson KC. COVID-19 interstitial pneumonia: monitoring the clinical course in survivors. Lancet Respir Med 2020; 8: 839-842

15 George PM, Barratt SL, Condliffe R, et al. Respiratory follow-up of patients with COVID-19 pneumonia. Thorax 2020; 75: 1009-1016

16 Graham BL, Steenbruggen I, Miller MR, et al. Standardization of spirometry 2019 update. An official American Thoracic Society and European Respiratory Society technical statement. Am J Respir Crit Care Med 2019; 200: e70-e88.

17 Graham BL, Brusasco V, Burgos F, et al. 2017 ERS/ATS standards for single-breath carbon monoxide uptake in the lung. Eur Respir J 2017; 49; 1600016.

18 Holland AE, Spruit MA, Troosters T, et al. An official European Respiratory Society/American Thoracic Society technical standard: field walking tests in chronic respiratory disease. Eur Respir J 2014; 44: 1428-1446.

19 Wanger J, Clausen JL, Coates A, et al. Standardisation of the measurement of lung volumes. Eur Respir J 2005; 26 : 511-522.

20 American Thoracic Society/European Respiratory Society. ATS/ERS statement on respiratory muscle testing. Am J Respir Crit Care Med 2002; 166: 518-624

21 World Health Organization (WHO). Clinical Management of COVID-19. WHO/2019-nCoV/clinical/2020.2015. www.who.int/publications/i/item/clinical-management-of-covid-19

22 Francone M, Iafrate F, Masci GM, et al. Chest CT score in COVID-19 patients: correlation with disease severity and short-term prognosis. Eur Radiol 2020; 30: 6808-6817.

23 von der Thüsen J, van der Eerden M. Histopathology and genetic susceptibility in COVID-19 pneumonia. Eur J Clin Invest 2020; 50: e13259.

24 Wang L, He W, Yu X, et al. Coronavirus disease 2019 in elderly patients: characteristics and prognostic factors based on 4-week follow-up. J Infect 2020; 80: 639-645.

25 Zhou F, Yu T, Du R, et al. Clinical course and risk factors for mortality of adult inpatients with COVID-19 in Wuhan, China: a retrospective cohort study. Lancet 2020; 395: 1054-1062.

26 Hussain A, Mahawar K, Xia Z, et al. Obesity and mortality of COVID-19. Meta-analysis. Obes Res Clin Pract 2020; 14: 295-300

27 Stefan N, Birkenfeld AL, Schulze MB, et al. Obesity and impaired metabolic health in patients with COVID-19. Nat Rev Endocrinol 2020; 16: 341-342.

28 Yang J, Hu J, Zhu C. Obesity aggravates COVID-19: a systematic review and meta-analysis. J Med Virol 2021; 93 : 257-261.

29 Herridge MS, Tansey CM, Matté A, et al. Functional disability 5 years after acute respiratory distress syndrome. $N$ Engl J Med 2011; 364: 1293-1304.

30 Ahmed H, Patel K, Greenwood DC, et al. Long-term clinical outcomes in survivors of severe acute respiratory syndrome and Middle East respiratory syndrome coronavirus outbreaks after hospitalisation or ICU admission: a systematic review and meta-analysis. J Rehabil Med 2020; 52: jrm00063.

31 Zhao YM, Shang YM, Song WB, et al. Follow-up study of the pulmonary function and related physiological characteristics of COVID-19 survivors three months after recovery. EClinicalMedicine 2020; 25: 100463

32 Zhou Z, Guo D, Li C, et al. Coronavirus disease 2019: initial chest CT findings. Eur Radiol 2020; 30: 4398-4406.

33 Wang H, Wei R, Rao G, et al. Characteristic CT findings distinguishing 2019 novel coronavirus disease (COVID-19) from influenza pneumonia. Eur Radiol 2020; 30: 4910-4917.

34 Xiong Y, Sun D, Liu Y, et al. Clinical and high-resolution CT features of the COVID-19 infection: comparison of the initial and follow-up changes. Invest Radiol 2020; 55: 332-339.

35 Cui N, Zou X, Xu L. Preliminary CT findings of coronavirus disease 2019 (COVID-19). Clin Imaging 2020; 65: $124-132$. 
36 Yu M, Liu Y, Xu D, et al. Prediction of the development of pulmonary fibrosis using serial thin-section CT and clinical features in patients discharged after treatment for COVID-19 pneumonia. Korean J Radiol 2020; 21: 746-755.

37 Ackermann M, Verleden SE, Kuehnel M, et al. Pulmonary vascular endothelialitis, thrombosis, and angiogenesis in Covid-19. N Engl J Med 2020; 383: 120-128.

38 Hosseiny M, Kooraki S, Gholamrezanezhad A, et al. Radiology perspective of coronavirus disease 2019 (COVID-19): lessons from severe acute respiratory syndrome and Middle East respiratory syndrome. AJR Am J Roentgenol 2020; 214: 1078-1082.

39 Luyt CE, Combes A, Becquemin MH, et al. Long-term outcomes of pandemic 2009 influenza A(H1N1)-associated severe ARDS. Chest 2012; 142: 583-592.

40 Ebner L, Funke-Chambour M, von Garnier C, et al. Imaging in the aftermath of COVID-19: what to expect. Eur Radiol 2020; in press [https://doi.org/10.1007/s00330-020-07465-6]. 